\title{
LA DOBLE RECEPCIÓN DEL SPINOZISMO EN ALEMANIA DURANTE EL SIGLO XVIII*
}

\author{
María Jimena Solé **
}

\section{Resumen}

Este artículo reconstruye la recepción del pensamiento de Spinoza en Alemania desde 1670 hasta 1789 y propone una nueva interpretación de este proceso. Sostenemos que la recepción del spinozismo en Alemania durante este periodo es controlado por una imagen de esta doctrina, que la presenta esencialmente como ateísta. Creemos que esta caracterización del spinozismo como un ateísmo recubre dos constelaciones problemáticas diferentes. Por un lado, se refiere a la incompatibilidad de la filosofía spinoziana con el teísmo y remite a los peligros que ésta representa para el orden religioso, moral y político basado en la existencia de un Dios teísta. El problema que subyace es, por lo tanto, la cuestión de los fundamentos metafísicos necesarios para sostener un determinado orden. Por otro lado, Spinoza es denunciado como un ateísta por su intención de demostrar racionalmente la existencia de Dios, por sostener que la razón humana es capaz de accede a la verdad sin auxilio externo, sin iluminación sobrenatural. Este segundo aspecto de la recepción del spinozismo se superpone con la discusión acerca de los alcances y límites de la razón humana y, por lo tanto, con el núcleo mismo de la Ilustración.

Palabras clave: Spinoza, Alemania, Siglo XVIII, Recepción, Discusión.

\section{Abstract}

This article reconstructs the reception of Spinọza's thought from 1670 up to 1789 in Germany and proposes a new interpretation of this process. We argue that the reception of Spinozism en Germany during this period is controlled by an image

" Una versión preliminar de este texto, "Einige Bemerkungen zur Spinoza-Rezeption in Deutschland", fue presentada y puesta en discusión en el Forschungskolloquium de la Leibniz Universität Hannover, Alemania, organizado por el Prof. Dr. Manfred Walther el 21 de noviembre de 2009.

** Doctora en Filosofía por la Universidad de Buenos Aires, donde también obtuvo los títulos de Licenciada en Filosofía y Profesora de Enseñanza Secundaria, Normal y Especial de Filosofía y donde se desempeña como Ayudante Docente en la Cátedra de Filosofía Moderna. Actualmente es Becaria Postdoctoral de CONICET. 
of this doctrine, which depicts it essentially as atheist. We think that the characterization of spinozism as atheism covers two different problematic constellations. On the one hand, it refers to the incompatibility of Spinoza's philosophy with theism and remits to the dangers that it represents for the religious, moral and political order based on the existence of a theistic God. The problem that underlies this fight against Spinoza is, therefore, the problem of the metaphysical foundation necessary to support a determined order. On the other hand, Spinoza is denounced as an atheist because of his intention to rationally demonstrate the existence of God, for believing that human reason is capable of accessing truth without external aid or supernatural illumination. This second aspect of the Spinoza-reception overlaps with the discussion about the competence and the boundaries of human reason and, therefore, with the principle of Enlightenment.

Keywords: Spinoza, Germany, $18^{\text {th }}$ Century, Reception, Discussion.

Es una opinión generalmente aceptada, que la recepción de la doctrina de Spinoza en Alemania desde la aparición del Tractatus theologico politicus [TTP] en 1670 y durante todo el siglo XVIII es un caso único en la historia de la filosofía. En ningún otro lugar del mundo Spinoza generó tanta atracción ni fue objeto de tantas polémicas y discusiones ${ }^{1}$. A lo largo de los ciento cincuenta años que siguen a la muerte de Spinoza, su doctrina fue interpretada por los pensadores alemanes de maneras muy diferentes, incluso opuestas e irreconciliables. Pero lo que vuelve este caso tan atrayente es que, durante ese periodo, parece haber una transformación radical en la valoración del spinozismo por parte de sus intérpretes. La violenta reacción inicial de rechazo a las ideas contenidas en el TTP dio lugar, durante las primeras décadas del reinado de Federico el Grande, a un abordaje

${ }^{1}$ Cfr. Baumgardt, David, "Spinoza und der deutsche Spinozismus", Kant-Studien, 32 (1927) p. 185; Altwicker, Norbert, "Spinoza. Tendenzen der Spinoza-Rezeption und -Kritik" en Idem., Texte zur Geschichte des Spinozismus, Darmstadt, Wissenschaftliche Buchgesellschaft, 1971, p. 2; Walther, Manfred, "Histoire des problèmes de la recherche" en Les Cahiers de Fontenay N. 36-38, Spinoza entre Lumières et Romantisme, Paris, 1985, p. 14. Testimonio de la gran curiosidad que despertaron las obras de Spinoza en este territorio es el hecho de allí fueron traducidas más rápido y más veces (véanse Walther, op. cit., p. 14 y Lagny, Anne, "Le spinozisme en Allemagne au XVIIIe siècle: Recherches actuelles" en Bloch, Olivier (comp), Spinoza au XVIIIe siecle, Paris, Méridiens Klincksieck, 1990, p. 288). 
filosófico del spinozismo junto a una tibia rehabilitación de su figura; esta actitud aparentemente más benévola finalmente dio paso, en el contexto de la denominada "polémica del spinozismo" a finales del siglo XVIII, a la reivindicación de Spinoza como un ejemplo moral y de su doctrina como filosóficamente valiosa.

La cuestión de la recepción del spinozismo en Alemania ha sido, pues, el objeto de numerosas investigaciones que intentan ofrecer una reconstrucción conceptual de este proceso $^{2}$. Entre aquellos que se han ocupado del problema de la recepción del spinozismo a lo largo del siglo XVIII de manera global y han ofrecido alguna hipótesis interpretativa, existe una doble tendencia. Por un lado, abordan este proceso en función de una división en diferentes etapas según la reacción predominante hacia su doctrina. Suele distinguirse un primer período a principios del siglo, en que Spinoza es atacado por sus adversarios y tergiversado por sus partidarios; y una segunda etapa, a finales del siglo, cuando la filosofía spinoziana es

2 Algunas de las obras centrales en la investigación sobre este tema son: Grunwald, Max, Spinoza in Deutschland, Aalen, Scientia Verlag, 1986; Timm, Hermann, Gott und die Freibeit. Studien zur Religionsphilosophie der Goethezeit.I: Die Spinozarenaissance, Frankfurt am Main, Vittorio Klostermann, 1974 ; Bell, David, Spinoza In Germany From 1670 To The Age Of Goethe, Institute of Germanic Studies, University of London, 1984; Schröder, Winifried, Spinoza in der deutschen Frübauflelärung, Würzburg, Königshausen \& Neumann, 1987; Zac, Sylvain, Spinoza en Allemagne. Mendelssobn, Lessing et Jacobi, Paris, Méridiens Klincksieck, 1989; Otto, Rudiger, Studien zur Spinozarezeption in Deutschland im 18. Jabrbundert, Frankfurt a.M., Peter Lang, 1994; Pätzold, Detlev, Spinoza - Aufklärung - Idealismus. Die Substanz der Moderne, Assen, Koninklijke van Gorcum, 2002. A excepción de León Dujovne, quien dedica dos capítulos del cuarto tomo de su obra sobre Spinoza a brindar algunos datos generales acerca de su recepción e influencia en Alemania, ningún autor de habla hispana, hasta donde sabemos, se ha dedicado a estudiar específicamente este asunto (cfr. Dujovne, León, Spinoza. Su vida, su época, su obra, su influencia, Buenos Aires, Losada, 1945, cap. V: "El espinosismo en Alemania hasta fines del siglo XVIII, pp. 97 a 113 y cap. VI: "El resurgimiento de Spinoza en Alemania desde Lessing hasta Hegel”, pp. 115 a 179). 
aceptada, exaltada y elogiada por los representantes de las nuevas corrientes de pensamiento ${ }^{3}$.

Por otro lado, los especialistas explican las distintas valoraciones del spinozimo a partir de la modificación que progresivamente se da en el contexto filosófico-teológico y/o en la cosmovisión dominante en cada una de estas etapas. En cuanto a las razones que estos autores ofrecen para explicar el tránsito de una etapa a la siguiente, éstas no son contradictorias ni entran en pugna unas con otras. Leo Bäck ${ }^{4}$, por caso, sostiene que hacia finales del siglo XVIII, la filosofía leibniz-wolffiana había perdido su poder y que esto contribuyó a que el spinozismo comenzara a ser valorado por primera vez de modo desprejuiciado. Spinoza ofrecía una nueva noción de divinidad en un momento en el que los hombres empezaban a alejarse de las opiniones religiosas tradicionales y además ofrecía un nuevo concepto de Naturaleza, al que podían adherir los pensadores románticos que profesaban una especie de espiritualismo natural. Complementando esta posición, Altwicker sostiene que fue la creciente emancipación del pensamiento filosófico de las tutelas teológicas y políticas, lo que permitió nuevas formas de pensar y despertó intereses teoréticos que no sólo contribuyeron a la rehabilitación histórica de Spinoza sino incluso a su utilización como un medio para legitimar las propias posiciones ${ }^{5}$. Teniendo en cuenta estas

${ }^{3}$ Véase Krakauer, Moses, Zur Geschichte des Spinozismus in Deutschland wäbrend der ersten Hälfte des 18. Jabrbunderts, Breslau, Schottlaender, 1881, p. 5; Grunwald, op. cit., p. 18; Altwicker, op. cit., pp. 3-4. Manfred Walther divide la historia de la recepción de Spinoza en tres fases. Primero, una etapa de total rechazo de Spinoza que va desde la primera reacción contra el Tratado teológico politico por parte de Jakob Thomasius en 1670 hasta Kant; una segunda fase, que va de Lessing hasta Hegel, en la que se aceptan algunos aspectos de la doctrina spinoziana y una tercera fase, que desemboca en el monismo de la filosofía positivista del siglo XIX y el nihilismo de Nietzsche, que reivindica al spinozismo. Walther aclara que la división en etapas no significa que no haya una superposición temporal entre ellas y que más bien representan las diferentes constelaciones conceptuales que, sucesivamente, han dominado la conciencia de cada época y que determinan la reacción que cada una tuvo frente a la doctrina de Spinoza (cfr. Walther, op. cit., p. 17).

${ }^{4}$ Cfr. Bäck, Leo, Spinozas erte Einwirkungen auf Deutscbland, Berlin, Mayer \& Müller, 1895, p. 85.

${ }^{5}$ Cfr. Altwicker, op. cit., p. 3. 
contribuciones, Walther sostiene que la recepción de Spinoza en Alemania ha sido determinada por el hecho de que su filosofía opera la negación radical de ciertos elementos que se encuentran en el centro del pensamiento filosófico tradicional, en particular, la noción de un Dios extramundano que crea al hombre y al mundo ex nibilo, la concepción de una moral basada en los mandamientos divinos exteriores al hombre y la idea de una economía divina de la salvación, de una providencia ${ }^{6}$. Las tesis interpretativas sostenidas por investigaciones más recientes, como las de W. Schröder o R. Otto, no contradicen estas hipótesis ni proponen una estructuración del tema diferente, sino que las ratifican o se concentran en especificar alguno de sus aspectos, apuntando principalmente al hecho de que el spinozismo era inaceptable para una sociedad regida por la ideología cristiana ${ }^{7}$.

Creemos que este modo de abordar la reconstrucción conceptual del proceso de recepción del spinozismo en Alemania presenta dos dificultades. Es cierto que desde 1670 y durante las primeras décadas del siglo XVIII existe una actitud generalizada de denuncia, crítica y rechazo del spinozismo, y que, hacia finales de ese siglo y principios del XIX, ésta se transforma en la actitud opuesta. Sin embargo, creemos que un análisis más detallado muestra que a lo largo de todo este período, Spinoza encuentra tanto amigos como enemigos entre sus lectores alemanes. A esto se añade una segunda dificultad.

Al estructurar la reconstrucción del proceso de recepción del spinozismo en Alemania en función de la valoración positiva o negativa hacia esta doctrina por parte de la mayoría pensadores alemanes en cada momento, se acepta, aunque sea tácitamente, el supuesto de que Spinoza y el spinozismo significaron lo mismo para los diferentes protagonistas de su

${ }^{6}$ Cfr. Walther, op. cit., p. 16.

7 Winfried Schröder, por ejemplo, establece que el rechazo y el combate que durante la primera mitad del siglo XVIII se dirige contra Spinoza se explica por el hecho evidente, a partir de sus obras, de que su doctrina era inaceptable en una sociedad cristiana (cfr. Schröder, op. cit., p. 15). Rüdiger Otto afirma, en el mismo sentido, que un análisis de la reacción anti-spinozista a lo largo del siglo XVIII pone de manifiesto que Spinoza era comprendido, al mismo tiempo, como un fenómeno singular y como el representante de una amenaza de las convicciones tradicionales cristianas (cfr. Otto, op. cit., p. 17). 
recepción y que, según distintos factores filosóficos, religiosos o ideológicos, éstos reaccionan de determinado modo frente a ella. Sin embargo, un recorrido por los textos que abordan la doctrina spinoziana desde finales del siglo XVII y durante todo el siglo XVIII pone en evidencia que el término "spinozismo" no refirió unívocamente a un mismo conjunto de ideas para sus lectores. Por lo tanto, para explicar las diferentes reacciones de los filósofos alemanes frente a Spinoza no es suficiente examinar hasta qué punto éstos estaban comprometidos con la ortodoxia cristiana o con la filosofía leibniziana.

Para comprender el proceso de la recepción de Spinoza en Alemania, dilucidar las diferentes actitudes hacia su doctrina por parte de los filósofos alemanes y revelar la importancia del spinozismo en el desarrollo de la filosofía alemana del siglo XVIII creemos que es necesario investigar, en primer lugar, de qué modo cada uno de ellos entendió esta doctrina en el contexto de sus propias convicciones filosóficas. En consecuencia, en vez de preguntar cuál es la actitud adoptada frente al spinozismo en los diferentes momentos del proceso de su recepción, preferimos estructurar esta investigación a través de la pregunta acerca de qué es lo que se discute cuando se discute acerca del spinozismo en Alemania. Esta primera pregunta nos conduce a atender a un hecho innegable: que la recepción de Spinoza en Alemania durante todo el siglo XVIII se realiza principalmente de un modo indirecto y que es en gran medida dominada por una imagen de Spinoza y del spinozismo se forjaba a través de rumores y escritos polémicos.

\section{La imagen de Spinoza}

En primer lugar, los especialistas aceptan que la recepción alemana de Spinoza no siempre estuvo ligada a la lectura directa de sus textos ${ }^{8}$. Dada la dificultad de conseguir sus libros -especialmente las Opera Posthuma [OP]- y dada la dificultad de acceder a la hermética doctrina de la Etbica, los pensadores alemanes de finales del siglo XVII y del XVIII a menudo accedieron a sus ideas a través de exposiciones fragmentarias y tendenciosas, que apuntaban únicamente a refutar y difamar al spinozismo.

${ }^{8}$ Cfr. Schröder, op. cit., p. 23; Otto, op. cit., p. 25. 
Muchas de esas críticas pronto se transformaron en lugares comunes y fueron repetidas y difundidas hasta el hartazgo. Poco a poco surgió una imagen de Spinoza que, aunque basada en ciertos elementos auténticos, no era sino una simplificación y deformación de su verdadero sistema, que, como se sigue de las breves referencias que acabamos de dar, incluso contenía elementos difíciles de conjugar entre sí.

El testimonio que Johann Christian Edelmann ofrece acerca de su primer contacto con el TTP pone en evidencia hasta qué punto esta imagen difundida entre los hombres de letras impedía un abordaje objetivo a sus textos. En su Autobiografía, Edelmann relata su reacción al recibir, en 1740, una encomienda de libros que le envía un amigo desde Berlín:

El primer [libro] que atrajo mi atención con una fuerte conmoción interior, fue el Tratado teológico politico de Spinoza; el atroz retrato que mis profesores me habían transmitido de este libro y de su autor quizás podría haberme conducido a dejarlo de lado sin haberlo leído, si yo no hubiese sabido por experiencia que lo mejor se encontraba en todos los escritos contra los cuales estos Señores solían advertir más fuertemente?.

Ya desvinculado de la ortodoxia de sus maestros, Edelmann decide leer el infame libro de Spinoza, cuyo solo nombre, según sus propias palabras, lo había "hecho estremecer durante sus años de estudiante"10.

La imagen de Spinoza que había hecho temblar a los hombres del siglo XVIII alemán es presentada elocuentemente por Herder, en un texto publicado en 1787. Allí dice:

Spinoza ha sido un ateo y panteísta, defensor de la doctrina de la absoluta necesidad, enemigo de la revelación, detractor de la religión y, por lo tanto, una amenaza para el Estado y la sociedad civil, en definitiva, ha sido un enemigo del género humano y como tal ha muerto. Se merece, por lo

${ }^{9}$ Edelmann, J.C., Selbstbiographie, Berlin, Klose, 1849, p. 350.

10 Ibid., p. 350. 
tanto, el odio y el asco de todo aquél que sea amigo del género humano y verdadero filósofo ${ }^{11}$.

Esta imagen compleja de Spinoza presenta, en primer lugar, un elemento que la mayoría de sus críticos -e incluso algunos de sus defensores- estuvieron de acuerdo en adjudicarle: el ateísmo.

La construcción de la imagen de Spinoza como un ateo comienza a gestarse desde su juventud. Expulsado de la sinagoga y sin adherir a ninguna religión, Spinoza pronto es identificado como un ateo y como un peligro para la paz del Estado holandés. Diferentes documentos confirman que hacia 1661, antes de haber publicado ninguno de sus escritos, Spinoza ya era conocido como un ateo en Holanda (Archivo Histórico de Madrid, Diario de viaje de Olaus Borch, documento de los habitantes de Voorburg). La difusión del rumor de que él identifica a Dios con la naturaleza confirmó y alimentó su fama. En parte para defenderse de tal acusación, Spinoza decide escribir el TTP, que empeora su situación al punto de que se le impide dar a la imprenta en manuscrito de la Ética, pues se había extendido el rumor de que su libro contenía una demostración de la no existencia de Dios ${ }^{12}$.

En Alemania, tanto los primeros críticos del TTP y las OP (J. Thomasius, Rappapolt, Musäus, Ch. Kortholt, Wachter) y los biógrafos de finales del silgo XVII (S. Kortholt, Bayle, Colerus, Stolle), como los representantes de la tempana Ilustración alemana (Leibniz, Ch. Thomasius, Wolff) y los protagonistas de la Polémica del spinozismo (Jacobi, Mendelssohn, Kant) vieron en el spinozismo un ateísmo y lo combatieron en tanto tal.

${ }^{11}$ Herder, Johann Gottfried, Gott. Einige Gespräche, 1787. Citamos según la edición crítica: Herder, J. G., Werke, Frankfurt am Main, Deutscher Klassiker Verlag, 1993, t. V, p. 683. La traducción al castellano es nuestra.

12 Sin embargo, también entre sus contemporáneos encontramos ciertos pensadores dispuestos a defender a Spinoza de tal acusación. En su Prefacio a las Opera posthuma Jarrig Jelles realiza una apología de Spinoza e intenta mostrar que la doctrina spinoziana no se contrapone al cristianismo (cfr. Jarig Jelles, Prafatio en B.d.S., Opera Posthuma, Amsterdam, Jan Rieuwertsz, 1677; trad. cast: en Domínguez, Atilano (ed.), Biografias de Spinoza, Madrid, Alianza, 1995, pp. 45-79). 
Con el tiempo, spinozismo, panteismo y ateismo devinieron sinónimos. En un libro publicado en 1701, titulado Exercitatio histórico-philosophica de Spinozismo ante Spinozam, J. F. Buddeus, teólogo luterano de Jena, utiliza por primera vez el término técnico Spinozismus para referirse a una forma peculiar de ateísmo ${ }^{13}$. Algunos años más tarde publica sus Lebr-Sätze von der Atheisterey und dem Aberglauben, en donde afirma que Spinoza fue el primero en asentar sus ideas impías de forma sistemática y, por eso, lo llama el Princeps Atheorum ${ }^{14}$. Además, Buddeus fue el primero en aplicar a Spinoza el término acuñado por Toland, calificándolo como un panteísta $a^{15}$, que él consideró sinónimo de la negación de la existencia de Dios y conectó con la tendencia mística y entusiasta a la que ésta doctrina podía servir de fundamento.

Pronto el término spinozista se transformó en una acusación. En el mismo texto al que hacíamos referencia hace un momento, Herder señala que "todos los impíos y ateos eran denominados spinozistas y en parte lo son aún"16. Ahora bien, la caracterización del spinozismo como un ateísmo contribuye a complicar la historia de la recepción de esta doctrina. Esto es así porque el concepto de ateísmo es en sí mismo complejo y a lo largo de la historia, se ha utilizado en diferentes sentidos y se ha aplicado a diferentes fenómenos y teorías ${ }^{17}$.

Tomado en su sentido más habitual, lo que podríamos llamar su uso vulgar, por ateísmo se entiende la negación de la existencia de Dios. Sin

13 Véase Sparn, "Formalis Atheus? Die Krise der protestantischen Orthodoxie gespiegelt in ihrer Auseinandersetzung mit Spinoza" en Gründer, K. y SchmidtBiggermann, W. (comps.), Spinoza in der Frübzeit seiner religiösen Wirkung, Heidelberg, Lambert Schneider, 1984, pp. 47 y ss.

${ }^{14}$ Buddeus, J., Lebr-Sätze von der Atheisterey und dem Aberglauben, Halle, 1717, p. 163.

${ }^{15}$ Ibid., p. 165. Véanse Otto, op. cit., pp. 64-66 y Pätzold, op. cit., p. 20. El término pantheist fue utilizado por primera vez por el filósofo John Toland en 1705, en su obra Socinianism Truly Stated para referirse a aquel que sostiene la posición según la cual Dios y el mundo son la misma cosa.

${ }^{16}$ Herder, op. cit., p. 683.

17 Véase Mauthner, Fritz, Der Atheismus und seine Geschichte im Abendlande, Stuttgart, Deutsche-Verlag, 1922 (reimpr.: Hildesheim/Zürich/New York, Georg Olms Verlag, 1985). 
embargo, esta negación puede realizarse de diferentes modos: de manera teórica o práctico-existencial; y si es teórica, con mayor o menor fundamentación en razones ${ }^{18}$. Pero en cuanto al sentido más específico de este concepto, hay que admitir que el a-teísmo es esencialmente un fenómeno reactivo y relativo. Se trata de la negación de una posición determinada: el teísmo. Juzgar una doctrina como ateísta o a una persona como atea depende, pues, de considerar que sus convicciones son opuestas a las usualmente atribuidas al teísmo, esto es, la postulación de un Dios trascendente, personal, libre, dotado de entendimiento y creador del universo, que se revela a los hombres.

Frente a un ateísmo que podría ser considerado absoluto, por negar la posibilidad de que exista una divinidad en general y, con ella la posibilidad de una religiosidad o dimensión espiritual en el hombre, el ateísmo en sentido estricto únicamente se opone a la existencia del Dios teísta, dejando abierta la posibilidad a que exista alguna otra clase de divinidad.

La identificación del spinozismo como un ateísmo transfirió toda la ambigüedad de este segundo concepto a la doctrina de Spinoza. Efectivamente, al observar la historia de la recepción de Spinoza en Alemania, se comprueba que éste es acusado de ateísmo en casi todos los sentidos que este concepto recubre. Incluso quienes negaron que el spinozismo fuera una doctrina ateísta contribuyeron a la confusión, pues utilizaron el término con significados muy distintos.

De esta manera, el análisis de la imagen de Spinoza que determina el carácter de su recepción en Alemania conduce a investigar el sentido en que su doctrina fue considerada como un ateísmo -así como el sentido en los que no fue considerada como tal- por parte de sus lectores alemanes. Esta indagación parece prometer poner en evidencia qué significó esta doctrina para cada uno de ellos y, a su vez, comenzar a comprender la reacción de cada uno de ellos frente al spinozismo. A su vez, conduce al esclarecimiento de qué es lo que se discute, cuando se discute acerca del spinozismo en

18 Para un análisis del concepto de ateísmo, véase Wucherer, Augustinus Karl, "Phänomen und Bedeutung des gegenwärtigen Atheismus. Philosphischtheologische Analyse und Sinndeutung" en A. K. Wuchererer, J. Figl y S. Mühlberger, Weltphänomen Atheismus, Wien, Herder, 1979, pp. 37-39. 
Alemania y, de este modo, a comprender el proceso de su recepción por parte de los pensadores alemanes en toda su complejidad.

Así pues, a continuación reconstruiremos conceptualmente el proceso de recepción de Spinoza por parte de los pensadores alemanes desde finales del siglo XVII hasta el Spinozismusstreit tomando como hilo conductor el concepto de ateísmo y procurando analizar, en cada caso, qué sentido se le atribuyó a este término al aplicarlo al spinozismo. Mostraremos que existen principalmente dos sentidos en los que la doctrina spinoziana es considerada de este modo y que éstas remiten a dos constelaciones problemáticas diferentes, que, según nuestra propuesta, podrían considerarse como dos niveles diferentes, aunque conectados, en los que se desarrolla la recepción de Spinoza en Alemania.

\section{Primera recepción de Spinoza en Alemania}

El TTP, publicado en 1760, generó en el territorio alemán una violenta reacción, especialmente en los ámbitos universitarios. La crítica a la revelación bíblica y al orden monárquico, la defensa de la libertad de pensamiento y expresión, la propuesta de la tolerancia religiosa y la separación entre la Iglesia y el Estado iban contra los fundamentos del orden religioso y político vigente en el territorio alemán. Así pues, desde 1670 y durante las últimas décadas del siglo XVII surgieron numerosos escritos que analizan y refutan, con diferente pretensión de objetividad filosófica, pero con igual dureza y violencia, las tesis principales del TTP. J. Thomasius, Dürr, Müller, Musaeus, Ch. Kortholt, Webber y Kettner son los principales representantes de esta reacción ${ }^{19}$. Estos escritos intentan poner

19 Véase Thomasius, Jacob, "Programma adversus anonymum, de libertate philosophandi" (1670) en Idem., Dissiertationes LXIII varii argumenti, Halle, Zeitler, 1693; Rappolt, Friedrich, Oratio contra naturalistas, en Idem., Opera Theologica, Leipzig, 1693; Dürr, Johann Conrad, Oratio de praepostera et impia libetate philosophandi, praesertim in religionis negotio, oppositam Tractatui Theologico-Politico scriptoris lucifugae haud ita pridem vulgato. Accessit ejusdem argumenti Programma Lipsiense Jacobi Thomasii, S.E. Buchta, Jena, 1672; Müller, Johannes, Atheismus devictus, Hamburg, 1672; Musaeus, Johannes, Spinozismus, boc est Tractatus theologico politicus, quo auctor quidam anonymus, conatu improbo, demostratum ivit, libertatem philosophandi, Literis Bauhoferianis, Jena, 1674; Kortholt, Christian, De tribus impostoribus Magnus liber, Kiel, Reumann, 1680.; Kettner, De duobus 
en evidencia el peligro práctico que representa el spinozismo para el orden religioso y político. Al hacerlo, recurren a un tono sumamente violento, a insultos y burlas: el TTP es presentado, por ejemplo, como "pessimae notae liber" 20 y Spinoza como un formalis atheus ${ }^{21}$, un enviado del diablo con la misión de subvertir los derechos divinos y bumanos para perjuicio de la Iglesia y el Estado ${ }^{22}$, como alguien que debería haberse dado el nombre de Maledictus Spinoza ${ }^{23}$. Su estrategia argumental es mostrar que la tesis central del TTP que las propuestas políticas que allí se defienden no amenazan sino que salvaguardan la paz pública- es falsa.

La denuncia de ateísmo contra Spinoza hecha por estos pensadores apunta, pues, principalmente a señalar que lo peligrosas que son las consecuencias prácticas de esta doctrina. En este sentido, la reacción contra el spinozismo se superpone con el combate de otras posiciones y doctrinas igualmente escandalosas, como el materialismo de Hobbes, Herbert de Cherbury o Matthias Knutzen.

Esta rápida identificación por parte de los profesores universitarios del spinozismo como un ateísmo peligroso explica en cierta medida la relación de Leibniz con esta doctrina. A pesar de admitir que existen ciertas similitudes entre sus sistemas, el alemán se esfuerza por distanciarse tanto personal como doctrinalmente de Spinoza. Y si bien no duda en considerarlo un ateo por haber negado la existencia de un Dios trascendente y un fatalista por haber negado la existencia de causas finales, Leibniz

impostoribus, Leipzig, 1694; Webber, Emmanuel, Beurtheilung der Atheisterey Wie auch derer mebrsten deßhalben berüchtigsten Scbrifften, Leipzig, 1697.

20) Rappolt, op. cit., t. II, p. 2162 (cfr. Otto, op. cit., p. 27, nota 52).

${ }^{21}$ Thomasius, J., op. cit., p. 571.

${ }^{22}$ Musaeus, op. cit. (citado por Colerus, reproducido en Freudenthal, J./Walther, M., Die Lebensgeschichte Spinozas. Lebensbeschreibungen und Dokumente. Stark erveiterte und neu kommentierte Neuausgabe der Lebensgeschichte Freudenthal 1899, 2 tomos, StuttgartBad Cannstatt, Frommann-Holzboog, 2006, t. I., p. 150).

${ }^{23}$ Kortholt, Ch., op. cit., p. 139. 
atribuye estos "errores" que él mismo no cree necesario refutar, al hecho de que el spinozismo no es sino un cartesianismo exagerado ${ }^{24}$.

El problema del spinozismo como un racionalismo radicalizado es el núcleo de la polémica que, según creemos, puede ser considerada la primera "polémica del spinozismo" en territorio alemán. En el número de marzo de 1688 de la revista editada por él, Monatsgespräche, Thomasius hace referencia al TTP. Afirma que se trata de un texto que niega a Dios e indica que afortunadamente ya ha sido refutado por muchos hombres importantes. Pasa entonces a hablar de la Ética, explica su método y describe sus partes para concluir que "la primera parte es la más peligrosa, porque las otras se fundan en ella, pero es precisamente su Atheisterey lo que conservan" 25 . La raíz del ateísmo spinoziano ha de descubrirse en la incorrecta utilización por parte de Spinoza de la definición de sustancia. Esto es lo que, según este autor, conduce a la insensata identificación de Dios con la totalidad de lo creado y la consiguiente negación de la existencia de un Dios trascendente, personal y creador. En ese mismo número de su revista mensual, Thomasius incluye una reseña del libro Medicina mentis de Walther von Tschirnhaus, publicado en Amsterdam el año anterior, cuyo fin era denunciarlo por contener ciertas tesis spinozistas; particularmente, la propuesta de fundamentar una ética sin recurrir a la revelación.

A pesar de que había sido discípulo de Spinoza y de que, efectivamente, existen evidencias de la influencia de aquél sobre su texto, es claro que Tschirnhaus deseaba evitar el peligro de ser asociado a una doctrina tan difamada. Se defendió en un escrito que envió a Thomasius en forma privada, que Thomasius publicó en el número de junio de los Monatgespräche junto con su propia réplica, donde reitera su acusación.

${ }^{24}$ En el $§ 393$ de sus Essais de Teodicée Leibniz presenta al spinozismo como "un Cartesianisme outre" (Leibniz, G.W., Die philosophischen Schriften von Gottfried Wilhelm Leibniz, ed. C.I. Gerhardt, Berlin, Weidmann, 1875-1887, t. VI, p. 350).

${ }_{25}$ Thomasius, Ch., Freimütige, lustige und ernsthafte, jedoch vernunftmässige Gedanken oder Monatsgespräche über allerband, fürnebmlich aber neue Bücher, tomo 1: enero a junio de 1688. (Reproducción de la edición de Halle, 1690), Frankfurt am Main, Athenäum, 1972, pp. 340-341. 
Un texto publicado algunos años más tarde, evidencia qué entiende Thomasius por ateísmo. "El ateo cree que él es más noble que las otras criaturas que lo rodean y no honra a nadie, no ama a nadie, no confía en nadie ni teme a nadie más que a sí mismo" 26 , escribe allí. Por consiguiente, sostiene, si un ateo se comporta del mismo modo que lo hacen los hombres creyentes, y "hace todo aquello que realiza un hombre virtuoso, quien busca o posee la mayor dicha y felicidad" es por motivos muy diferentes. El ateo lo hace porque su razón le enseña que llevar una "vida irracional" lo haría muy infeliz ${ }^{27}$. Así, reemplaza el temor de Dios por su propia razón.

El problema al que apunta este ataque contra los ateos no consiste, pues, en si éstos son elementos nocivos para la paz del Estado y la vida en sociedad. Su comportamiento exterior, en tanto que imitaría al de los otros hombres que sí creen en Dios y aceptan sus leyes, no pone en peligro el orden. Al contrario, el problema al que Thomasius apunta es el de denunciar a la razón como una facultad que, al pretender ser la fuente de la moral, en vez de elevar a los hombres, los reduce a animales que meramente imitan a los que sí pueden sentir el temor de Dios.

La polémica entre Thomasius y Thschirnhaus tiene como trasfondo este mismo problema. Thomasius afirma que el núcleo filosófico del spinozismo es la posición según la cual la razón puede demostrarlo todo y fundamentar incluso una moral. $Y$ es por esto que lo considera un ateísmo. Así pues, en el marco de esta polémica, el término "spinozist" es utilizado por primera vez como una acusación, remitiendo a la posición según la cual el entendimiento existe en los seres humanos tal como existe en Dios ${ }^{28}$.

El interés que despertaba la doctrina de Spinoza en Alemania explica el hecho de que, en el cambio de siglo, dos alemanes -el profesor de literatura Sebastian Kortholt y el pastor luterano Johannes Colerus- realizan investigaciones en Holanda acerca de la vida y la obra de Spinoza que

26 Thomasius, Ch., Einleitung zur Sittenlebre, Halle, 1692, p. 149 (cfr. CzelinskyUesbeck, Michael, Der tugendhafte Atheist. Studien zur Vorgeschichte der SpinozaRenaissance in Deutscbland, Würzburg, Könighasen u. Neumann, 2007, p. 137).

${ }^{27}$ Ibidem.

${ }^{28}$ Thomasius, Ch. Freimütige, lustige und ernsthafte, jedocb vernunftmässige Gedanken oder Monatsgespräche, ed. cit., p. 841. 
plasman en sendos estudios biográficos ${ }^{29}$. Junto con el artículo incluido en el Diccionario bistórico-crítico de Pierre Bayle, que aparece por primera vez en 1697, estos textos tuvieron una gran difusión por todo el territorio alemán ${ }^{30}$.

Bayle, Colerus y S. Kortholt contribuyen a terminar de forjar la fama de Spinoza como un ateo por haber propuesto una doctrina según la cual la naturaleza toda es identificada con la divinidad. Sin embargo estos textos, presentan una serie de paradojas: por un lado, el hecho de que Spinoza es retratado como un ateo virtuoso; por otro, el hecho de que su pensamiento es caracterizado como absurdo e inaceptable y, a la vez, como sistemático y fundado en razones.

Hasta finales del siglo XVIII estas tres biografías fueron la fuente principal de conocimiento del spinozismo en Alemania. Como ya adelantamos, sus críticas fueron repetidas y difundidas por otros escritos hasta transformarse en lugares comunes de la literatura anti-spinozistas. Pero las paradojas que presentan en conexión con la vida y con la doctrina de Spinoza, cumplen la función de generar aún más curiosidad en los lectores alemanes.

A esto último también contribuyen los textos de otros dos autores de origen alemán. Georg Wachter, quien afirma en una obra de 1699, que el spinozismo conduce a la divinización de la naturaleza y afirma que éste es

29 Colerus, Johannes, Korte dog waaragtige levensbeschriyving van Benedictus de Spinoza uit Autentique Stukken en mondeling getuigenis van noglevende Personen, opgestelt [Breve pero veraz biografia de Benedictus de Spinoza, recogida a partir de testimonios auténticos escritos y orales de personas aun vivas], Amsterdam, J. Kindenberg, 1705; Kortholt, Sebastian, "Praefatio" en Kortholt, Christian, De tribus impostoribus Magnus liber, Kiel, Reumann, 1700 ( $2^{\circ}$ ed.).

${ }^{30}$ Pierre, Dictionnaire bistorique et critique, Amsterdam/Leiden/La Haya/Utretch, P. Brunel, 1696-7. Su gran éxito motivó numerosas reediciones. Hacia 1740 se había reeditado siete veces. Para muchos pensadores del siglo XVIII, el artículo de Bayle fue la única fuente de datos acerca de la vida y doctrina de Spinoza. Al respecto, véase Bahr, Fernando, "Spinoza en el Dictionnaire de Pierre Bayle" en Tatián, D. y otros, Primer Coloquio Spinoza, Buenos Aires, Altamira, 2005. 
heredero de la filosofía mística de la Cábala judía, fuente de su ateísmo ${ }^{31}$. Pero contribuyendo a oscurecer aún más la imagen de Spinoza, algunos años más tarde Wachter se retracta y ensaya una interpretación emanacionista del spinozismo, sosteniendo que ni esta doctrina ni la cábala debían ser consideradas ateístas, pues aceptan y defienden la existencia de una divinidad ${ }^{32}$. Por su parte, Conrad Dippel, un decidido antiortodoxo, acusa al spinozismo, sin ahorrarse insulto alguno, de ser ateo por haber colocado como fundamento al diabólico Fatum, por haber abrazado un brutal fatalismo que, al negar la libertad en Dios y los hombres, vuelve imposible la moral ${ }^{33}$. Dippel, quien defiende un espiritualismo ético, sostiene que el pecado de Spinoza es un resultado de la aplicación de la razón a la investigación teológica y, sin embargo, reconoce en el racionalismo de Spinoza una gran coherencia y, por tanto, cierto valor ${ }^{34}$.

\section{El spinozismo en el nacimiento de la Ilustración alemana}

Durante las primeras décadas del siglo XVIII, estalla una segunda polémica en la que un filósofo alemán es acusado de spinozimo y, por lo tanto, de ateísmo. Se trata de Christian Wolff, perseguido por el teólogo J. Lange, su colega de la universidad de Halle, por haber sostenido la independencia de la moral respecto de la religión.

En 1721 Wolff fue nombrado rector de la Universidad y su discurso inaugural, titulado Oratio de Sinarum philosophia practica sostiene que los principios éticos son independientes de la creencia religiosa. Wolff afirma que la moral de los chinos se basa en el conocimiento, a través de la razón,

31 Cfr. Wachter, Georg, Der Spinozismus im Jüdenthumb oder, die von dem beütigen Jüdenthumb, und dessen Gebeimen Kabbala Vergötterte Welt [El spinozismo en el judaismo o el mundo divinizado/idolatrado por el judaismo actual y su Kabbala secreta], Amsterdam, 1699. ${ }^{32}$ Cfr. Wachter, Georg, Elucidarius Cabalisticus sive reconditae Hebraeorum philosophiae Brevis et succincta recensio, Roma, 1706.

${ }^{33}$ Cfr. Dippel, J.C., Fatum fatuum ..., en Eröffneter Weg zum Frieden mit GOTT und allen Creaturen, Durch die Publication der sämtlichen Schrifften Christiani Democriti, Berleburg, 1747, p. 69.

${ }^{34}$ Alexander, G., "Spinoza und Dippel” en Gründer, K. y Schmidt-Biggermann, W. (comps.), op. cit., p. 106. 
de las nociones de bien y de mal y que, por lo tanto, el ejercicio de la virtud depende de la investigación racional de la naturaleza ${ }^{35}$.

Reaccionando en su contra Joachim Lange publica, en 1723, un texto titulado Causa Dei et Religionis Naturalis Adversus Atheismum, donde se ocupa de analizar todos los sistemas que él considera que pueden llevar al ateísmo o que son en sí mismos ateístas. Según Lange, el ateísmo es el resultado de un intelecto corrupto y los sistemas que conducen a él deben ser considerados pseudophilosophia ${ }^{36}$. Mientras que, por ejemplo, la filosofía estoica y la doctrina de Hobbes son presentadas como conteniendo la causa del ateísmo y el sistema cartesiano como una doctrina que puede dar ocasión a este error, Lange afirma que el spinozismo es en sí mismo un sistema ateísta ${ }^{37}$. Este juicio se basa en el hecho de que, según él, Spinoza considera que Dios es el universo y que el universo es Dios además de que establece una absoluta necesidad sobre todas las cosas que elimina la noción de contingencia y anula la posibilidad de los milagros. De modo que el primer error de Spinoza reside, según Lange, en haber hecho de Dios la sustancia del mundo, es decir, en haberlo negado ${ }^{38}$. El segundo error es haber establecido, al igual que los estoicos, un "absoluto mechanismi fato" que implica la negación de la libertad tanto en Dios como en los hombres ${ }^{39}$.

Además de motivar su expulsión de la universidad y su destierro del reino de Prusia, esta acusación y la subsiguiente polémica obligan a Wolff a estudiar a Spinoza y a intentar diferenciarse de su posición. El resultado de este estudio es la refutación del sistema metafísico spinoziano que Wolff incluye en la segunda parte de su Theologia naturalis publicada en 1737. Este texto representa un verdadero hito en la historia de la recepción de Spinoza en Alemania, pues hasta entonces ningún pensador en el territorio alemán se había ocupado de analizar la teoría metafísica de Spinoza, tal como es expuesta en la Ética, de modo serio, filosófico y sistemático. Las críticas allí

35 Véase Wolff, Christian, Oratio de Sinarum philosophia practica/Rede iiber die praktische Philosopbie der Chinesen, Hamburg, Meiner, 1985.

${ }^{36}$ Cfr. Lange, Causa Dei et Religionis Naturalis Adversus Atheismum, Halle, 1723, pp. 32 y ss.

${ }^{37}$ Ibid., p. 54.

${ }^{38}$ Ibid., p. 55.

${ }^{39}$ Ibid. p. 56. 
expuestas, sin embargo, no son ni profundas ni exhaustivas. Principalmente, Wolff se dedica a analizar las definiciones de la Ética y a mostrar que son arbitrarias. Según su interpretación, es la definición de sustancia la que conduce a la afirmación del fatalismo. Su conclusión es que el spinozismo es aún más peligroso que el ateísmo, pues éste excluye la posibilidad de la moral al negar la libertad en Dios y los seres humanos ${ }^{40}$.

Durante las primeras décadas del siglo XVIII, surgen ciertos pensadores considerados spinozistas clandestinos, pues reivindican algunas de las tesis del TTP e invocan el nombre de Spinoza en su lucha contra la Iglesia luterana que consideraban corrupta. Hombres como Friedrich Wilhelm Stosch, Theodor Ludwig Lau y Johann Christian Edelmann encontraron en Spinoza a un aliado en muchas de sus causas políticas, como la reivindicación de la tolerancia religiosa y la libertad de conciencia, así como también en algunas de sus convicciones teológicas. En efecto, la doctrina spinoziana de la inmanencia apoyaba la idea de que cada individuo puede acceder directamente a Dios, sin necesidad de mediaciones externas. Spinoza no es, para ellos, un ateo en el sentido de negar absolutamente la existencia de Dios, sino que su doctrina les provee un concepto diferente de la divinidad que permite mantener una posición que ellos consideraron cristiana, aunque se alejara de la ortodoxia. Al igual que Wolff, todos ellos debieron sufrir las consecuencias de la acusación de spinozismo.

Por haber reivindicado el spinozismo explícitamente, Edelmann es quizás el representante más significativo entre estos pensadores clandestinos. En un texto publicado en 1740, este autor retoma la crítica spinoziana a las Sagradas Escrituras tal como aparece en el TTP y declara que prefiere ser ateo con Spinoza antes que cristiano con los ortodoxos ${ }^{41}$. Sin embargo, él no está dispuesto a considerar al spinozismo como un ateísmo, pues ve en la doctrina spinoziana la representación del Dios verdadero, del criterio de la religiosidad. Edelmann fue perseguido por las autoridades religiosas y civiles. Fue obligado a retractarse, sus libros fueron quemados públicamente y su fama como ateo hizo que fuese sucesivamente

40 Wolff, Ch., Theologia naturalis, Methodo scientifica pertractata, Frankfurt am Main/Leipzig, Regner, 1737, parte II, $₫ 716$.

${ }^{41}$ Edelmann, J.C., Moses mit aufgedeckten Angesichte [Moisés con el rostro descubierto], 1740, parte I, pp. 138-9. 
expulsado de las ciudades donde se instaló. Se ocultó en Berlín, donde murió olvidado.

Cuatro años después de la aparición del polémico texto de Edelmann -fecha que coincide además con la coronación de Federico el GrandeJohann Lorenz Schmidt traduce al alemán y publica la Ética de Spinoza. La inclusión de la refutación de Wolff, es lo que debe haber permitido que fuese aprobado por la censura. En efecto, el título del libro es La Ética de Spinoza refutada por el conocido filósofo de nuestro tiempo, el Sr. Chistian Wolff. Pero además, Schmidt incluye en el volumen el Prefacio de Jelles, que ofrece una apología del spinozismo, y un índice temático que permiten al lector abordar el texto más fácilmente.

A pesar de afirmar explícitamente, en el prefacio de la obra, que su intención es la de poner en evidencia que la doctrina spinoziana ya había sido suficientemente refutada ${ }^{42}$, Schmidt fue acusado de cripto-spinozista, buscado por la justicia y forzado a vivir en la clandestinidad, bajo un seudónimo. Pero su contribución a la recepción del spinozismo en Alemania es inmensa y usualmente pasada por alto ${ }^{43}$. La traducción alemana de la Ética fue, sin embargo, la condición material que posibilitó el acceso directo al texto a un público mucho más amplio y permitió la paulatina puesta en duda de la imagen generalizadamente aceptada de su doctrina.

\section{La Polémica del spinozismo y el Spinozarenaissance}

En 1755, Moses Mendelssohn publica sus Diálogos filosóficos (1755), que consisten principalmente en un análisis de la doctrina de Spinoza y constituyen un intento explícito por recuperar filosóficamente la figura de Spinoza ${ }^{44}$. Mendelssohn presenta a Spinoza como un hombre a quien la historia de la filosofía le había negado, por no haber sabido comprenderlo,

${ }^{42}$ Cfr. B.v.S. Sittenlebre widerleget von dem berübmten Weltweisen unserer Zeit Herrn Christian Wolff, trad. y ed. de Johann Lorenz Schmidt, Leipzig / Frankfurt a. Main, 1744, pp. 3-4.

43 Véase Goldenbaum, U., "Die erste deutshce Übersetzung derl Spinozaschen Ethik" en Delf, H., Schoeps, J., Walther, M. (comps.), Spinoza in der europäischen Geistesgescbichte, Berlin, Hentrich, 1994, p. 108).

${ }^{44}$ Mendelssohn, M., Philosophische Gespräche, Berlin, Voss, 1755. 
el lugar honorífico que se merecía. Su primera estrategia de rescate consiste en mostrar que Spinoza representa un momento necesario en el desarrollo de la filosofía racionalista, pues es quien permite la transición de Descartes a Leibniz. Además, Mendelssohn sostiene que, bien interpretado, el sistema metafísico spinoziano es totalmente compatible con el desarrollado por Leibniz e incluso lo señala como el descubridor de la armonía preestablecida $^{45}$. De este modo, la doctrina tan combatida no se opone sino que se armoniza con aquella que constituía el fundamento filosófico sobre el que se construía la Ilustración alemana así como con el orden moral, religioso y político basado en un Dios teísta. Mendelssohn ensaya, pues, una doble salvación de Spinoza, tanto respecto del valor filosófico mismo de su sistema como respecto de sus posibles consecuencias prácticas.

Algunos años más tarde, Lessing discute esta interpretación del spinozismo. En dos fragmentos enviados a Mendelssohn durante 1763, publicados póstumamente, Lessing denuncia como incorrecta su interpretación y parece situarse del lado de Spinoza al adoptar una ontología monista, difícilmente armonizable con el concepto teísta de la divinidad ${ }^{46}$. En efecto, el análisis de ciertos textos de Lessing permite afirmar que éste postuló la existencia de un Dios inmanente que, a diferencia de lo sostenido por los enemigos del spinozismo, le posibilitó construir una teoría moral conectada con una doctrina política coherente con su credo ilustrado. Esto se confirma en la última obra publicada por Lessing en vida: La educación del género bumano, cuyo $\$ 73$ despertaría en Jacobi la sospecha de que tal vez Lessing era un spinozista ${ }^{47}$.

45 Cfr. Mendelssohn, M., Gesammelte Schriften. Jubiläumsausgabe, ed. A. Altmann, Berlin, Akademie-Verlag, 1929 y ss. Reedición a partir de 1974: Stuttgart - Bad Cannstatt, Friedrich Frommann Verlag (Günther Holzboog), t.1, pp. 6 y ss.

46 Los dos fragmentos son: "Durch Spinoza ist Leibniz nur auf die Spur der vorberbestimmten Harmonie gekommen" ["A través de Spinoza, Leibniz dio sólo con la pista de la armonía preestablecida"] y "Über die Wirklicbkeit der Dinge außer Gott" ["Sobre la realidad de las cosas fuera de Dios"]. Ambos fueron publicados póstumos bajo el título Spinozisterey en Lessing, Karl Gotthelf, Gotthold Ephraim Lessings Leben, nebst seinem noch übrigen litterarischen nacblasse, Berlin, Vossische Buchhandlung, 1795, t. II pp. 164-169.

${ }^{47}$ Léssing, Die Erziehung des Menschengeschlechts, 1780. 
En efecto, el mismo año en que aparece este texto de Lessing, Jacobi lo visita en Wolfenbüttel y allí mantienen una conversación que puede ser considerada como el momento central del proceso de recepción de Spinoza en Alemania. Según el relato que algunos años más tarde hace Jacobi de esta conversación, Lessing le habría confesado que los conceptos ortodoxos de la divinidad no lo satisfacían, que era un partidario del hen kai pan [uno y todo] y que estaba convencido de que "no hay otra filosofía que la filosofía de Spinoza" 48 .

Jacobi utiliza esta afirmación de Lessing para iniciar, luego de la muerte de éste, en una polémica epistolar con Mendelssohn. Por un lado, su interés reside en mostrar que, tal como lo reconoce Lessing, toda la filosofía racionalista conduce al spinozismo, pues esta doctrina representa el producto más acabado y perfecto de la tendencia propia de los seres humanos a conocerlo y explicarlo todo mediante su razón ${ }^{49}$. El rigor y la severidad en la aplicación del principio nada proviene de la nada es el rasgo que caracteriza al spinozismo y la marca que confirma que su sistema representa la radicalización de la posición racionalista, comprometida con el principio de razón suficiente.

Jacobi sostiene que todo sistema especulativo debe aceptar el principio de razón suficiente. Este principio es el requisito necesario para poder afirmar el encadenamiento lógico y causal, sin lagunas ni interrupciones, de los entes reales. Es también la causa de que se pueda exigir a la razón humana que recorra ese encadenamiento con pasos continuos, sin permitirle dar ningún salto, sin que deje nada sin explicación ${ }^{50}$. Así pues, en el ámbito de la realidad, todo hecho en el universo spinoziano tiene una causa; en el ámbito del pensamiento, todo

48 Cfr. Friedrich Heinrich Jacobi's Werke, Köppen y Roth (ed.), Gerhard Fleischer, Leipzig, 1812-1825 (Reimpr: Darmstadt, Wissenschaftliche Buchgesellschaft, 1968), t. IV.1, p. 55.

${ }^{49}$ Cfr. ibid., t. IV.2, pp. 129 y ss.

50 Véanse Kahlefeld, Susan, Dialektik und Sprung in Jacobis Pbilosopbie, Würzburg, Königshausen u. Neumann, 2000 p. 31 y Schick, Stefan, Vermittelte Unmittelbarkeit. Jacobis «Salto mortale» als Konzept zur Aufhebung des Gegensatzes von Glaube und Spekulation in der intellektuellen Anschaunngl der Vernunft, Königshausen u. Neumann, Würzburg, 2006 , p. 45. 
concepto tiene una razón. No existe el vacío ni la generación a partir de la nada, así como no existe una verdad que no pueda ser deducida a partir de otra. Aceptar este esquema determinista propio de la explicación científica y extenderlo a todos los ámbitos de la realidad, es lo que conduce de modo necesario e indefectible, sostiene Jacobi, al ateísmo y al fatalismo ${ }^{51}$.

De modo que Spinoza es para él el filósofo más coherente de la historia, que ha llevado los principios del racionalismo a su radicalización y, con honestidad, ha aceptado sus consecuencias. Sin embargo, estas consecuencias son para Jacobi inaceptables, pues el fatalismo y el ateísmo implican la aniquilación de la religión, la moral y el orden político tal como Jacobi los desea y defiende. Y dado que el spinozismo no puede ser refutado mediante argumentos, Jacobi propone un abandono de la filosofía mediante un salto mortal que lo depositaría en el ámbito de la revelación y la $\mathrm{fe}^{52}$.

Luego de la publicación en 1785 de sus Cartas sobre la doctrina de Spinoza al Sr. Moses Mendelssobn ${ }^{53}$, la cuestión del supuesto spinozismo de Lessing se volvió un asunto de discusión pública y Spinoza se transformó, hacia finales del siglo XVIII, en el problema central de la discusión filosófica en Alemania y ninguno de los pensadores prominentes de la época puede quedar al margen ${ }^{54}$. Pero el problema del credo de Lessing y el de la correcta interpretación de Spinoza quedó, gracias a la agudeza de la argumentación jacobiana, explícitamente conectado con el problema de la legitimidad del

51 "Todos los caminos de la demostración conducen al fatalismo", sostiene Jacobi en la cuarta de sus tesis breves sobre Spinoza (Friedrich Heinrich Jacobi's Werke, ed. cit., t. IV.1 p. 223). Pero, según él, "El fatum anula necesariamente a Dios, y Dios al fatum" (ibid., p. 218).

${ }^{52}$ Cfr. ibid., p. 59.

${ }^{53}$ Jacobi, F. H., Über die Lebre des Spinoza in Briefen an den Herrn Moses Mendelssobn, Breslau, 1785.

${ }^{54}$ Sobre la Polémica del panteísmo o del spinozismo, véase Scholz, H. (comp.), Die Hauptschriften zum Pantheismusstreit zwischen Jacobi und Mendelssobn, Hartmut Spenner, Waltrop, 1916 (reimpresión: 2004); Timm, H., op. cit.; Bell, David, Spinoza In Germany From 1670 To The Age Of Goethe, Institute of Germanic Studies, University of London, 1984; Christ, Kurt, Jacobi und Mendelssobn, Würzburg, Königshausen u. Neumann, 1988; Tavoillot, H-P., Le Crépuscule des Lumières. Les documents de la Querelle du panthéisme. 1780-1789, Paris, CERF, 1996; Sylvain Zac, Spinoza en Allemagne. Mendelssobn, Lessing et Jacobi, Paris, Méridiens Klincksieck, 1989. 
proyecto ilustrado. Enfrentarse a la doctrina spinoziana significó enfrentarse al problema de los límites de la razón.

Frente a la publicación del texto de Jacobi, Mendelssohn reacciona con un escrito que consume sus últimas energías (pues pocos días después de concluirlo, muere) titulado $A$ los amigos de Lessing55, en el que sostiene que Lessing jamás pudo haber adherido al spinozismo tal como Jacobi lo entiende ${ }^{56}$. Pero además acusa a Jacobi de haber injuriado a la razón y lo considera un fanático, un Schwärmer que pretende convencer a todo el mundo de su credo irracionalista. Él mismo no cree que la Ilustración se encuentre amenazada por las afirmaciones jacobianas y sostiene que, cuando la especulación parece ir demasiado lejos y se encuentra en peligro de perder su rumbo, hay que orientarse según el sentido común $o$ sano entendimiento ${ }^{57}$.

La respuesta de Jacobi a las acusaciones de Mendelssohn es contundente. Frente a lo que él ve como una deshonestidad por parte de los ilustrados berlineses, quienes abandonan la búsqueda de la verdad si piensan que ésta contradice lo que ellos consideran que es racional, alaba a Spinoza como "Santo Benedicto", por haber permanecido fiel a sus principios y por haber aceptado sus consecuencias ${ }^{58}$. Respecto de la acusación de haber injuriado a la razón por afirmar que ésta no puede demostrar la existencia de Dios, Jacobi se limita a preguntar “¿Y Kant?”. En efecto, ya en su primera edición de la Crítica de la razón pura, aparecida pocos años antes, Kant afirma

55 Mendelssohn, M., An die Freunde Lessings. Ein Anbang zu Herrn Jacobi Briefwechsel über die Lebre des Spinoza, Berlin, 1786.

${ }^{56}$ En el libro que él mismo había publicado como resultado de su enfrentamiento con Jacobi titulado Horas matinales o lecciones acerca de la existencia de Dios, Mendelssohn había sostenido que Lessing podría haber adherido a un "spinozismo purificado" que coincidía con su primera interpretación de esta doctrina en clave leibniziana y que no tenía ninguna consecuencia negativa para la moral y la religión (cfr. Mendelssohn, M. Morgenstunden oder Vorlesungen über das Daseyn Gottes, Voss, Berlin, 1785 en Idem., Gesammelte Scbriften. Jubiläumsausgabe, ed. cit., t. 3.2, p. 104).

${ }^{57}$ Cfr. ibid., p. 81.

${ }^{58}$ F. H. Jacobi, Wider Mendelssobns Beschuldigungen, betreffend die Briefe über die Lebre des Spinoza en Friedrich Heinrich Jacobi's Werke, ed. cit., t. IV.2, p. 245. 
la imposibilidad de demostrar la existencia de Dios y la necesidad de poner límites a la razón en su uso especulativo ${ }^{59}$.

El ataque de Jacobi a la Ilustración y la imposibilidad de Mendelssohn de defenderla, obliga a Kant a intervenir. Lo hace en un artículo titulado "¿Qué significa orientarse en el pensamiento?", en el que define su posición en la polémica y expone su crítica a la doctrina de Spinoza. Sin dar evidencias de poseer un conocimiento profundo del spinozismo, su crítica se basa fundamentalmente en la identificación de esta doctrina con un dogmatismo. Spinoza es un dogmático, pues no ha realizado la crítica y por lo tanto se fía del método matemático en metafísica y pretende poder conocerlo y demostrarlo todo. En tanto que representante del dogmatismo, Kant denuncia a la filosofía de Spinoza como conducente tanto al ateísmo como al fanatismo, esto es, al abandono de la razón y como un peligro para la moral y el orden ${ }^{60}$. La lucha contra Spinoza -pero también contra Mendelssohn y contra Jacobi- coincide en Kant con la tarea de justificar la necesidad de la crítica, que coincide a su vez con la necesidad de difundir y poner en marcha el proceso de la auténtica Ilustración, tal como él la redefine en oposición a Mendelssohn y los ilustrados berlineses. Según Kant, la Ilustración no consiste en acumular conocimiento y saberes, sino que se trata de conocerse a sí mismo y atreverse a ser autónomo.

Así pues, si el Spinozismusstreit pone al descubierto y profundiza la crisis de la metafísica moderna y de una Ilustración que confiaba en el absoluto poder de la razón demostrativa ${ }^{61}$, hay que admitir que esta polémica representa asimismo la coyuntura que permite que la Ilustración alemana renazca, triunfante y renovada de la mano de Kant.

59 Ibid., p. 255.

${ }^{60}$ Kant, I., "Was heißt: sich im Denken Orientieren?", Berlinische Monatsschrift, 1786 en Kants gesammelten Schriften, Akademieausgabe, Berlin, Preußischen Akademie der Wissenschaften, 1900 y ss, t. VIII, pp. 134 y ss.

${ }^{61}$ Cfr. Vaysse, J-M, Totalité et Subjectivité. Spinoza dans l'Idéalisme Allemand, Vrin, París, 1994, p. 10; Beiser, The Fate of Reason. German Philosophy from Kant to Fichte, Cambridge /London, Harvard University Press, 1987, p. 45; Tavoillot, op. cit., p. XI; Strauss, L., "Einleitung" en Mendclssohn, M., Gesammelte Schriften. Jubiläumsausgabe, ed. cit., t. 3.2, p. LX. 
Pero Jacobi no luchaba únicamente contra la Ilustración. También utilizaba a Spinoza para enfrentarse a Herder y Goethe, representantes de lo que él consideraba otra variante de ateísmo: el Sturm und Drang que proponía la adoración de la naturaleza y la celebración de la autonomía humana. Frente a las críticas que éste realiza a Spinoza, Herder y Goethe -quienes son impulsados por el debate entre Mendelssohn y Jacobi a retomar y profundizar el estudio de la Ética- se revelan como herederos de Lessing incluso tal vez de Edelmann- al adoptar con ciertas variaciones la noción spinoziana de Dios como un ser inmanente e impersonal, principio originario de toda vida, al que se descubre y conoce mediante la investigación científica de la naturaleza ${ }^{62}$. De este modo, combaten la idea de que el spinozismo represente un peligro para el orden y para la religión. $\mathrm{Al}$ contrario, ellos ven en esa doctrina la única capaz de ofrecer un concepto filosófico coherente de Dios, el hombre y la naturaleza. El 9 junio de 1785 Goethe escribe a Jacobi, expresando su total desacuerdo respecto de su valoración de la doctrina spinoziana y afirma que "si otros están dispuestos a llamar a Spinoza ateo, yo preferiría llamarlo y alabarlo como theissimum y christianissimum" 63 .

\section{La doble discusión en torno al spinozismo}

Podemos, entonces preguntarnos żqué es lo que se discute cuando se discute acerca del spinozismo en Alemania? A partir de la breve reconstrucción del derrotero de la doctrina spinoziana a lo largo de todo el siglo XVIII, creemos poder afirmar que esta doctrina enfrenta a los pensadores alemanes de todo el siglo XVIII a dos constelaciones problemáticas diferentes, que se conectan con los dos sentidos principales a los que remite la acusación de ateísmo arrojada contra ella.

${ }^{62} \mathrm{La}$ recepción de Spinoza por parte de Goethe se encuentra a lo largo de toda su obra. El texto que surge específicamente en el contexto del Spinozismusstreit se titulo "Studie nach Spinoza" (Goethe, Berliner Ausgabe, cd. Seidel, Berlin, Aufbau, 1960 y ss., t. 18, p. 140). Herder participa con su texto titulado Gott, einige Gespräche [Dios, algunas conversaciones], Gotha, Ettinger, 1787.

${ }^{6.3}$ Jacobi, F.H., Briefwechsel. Gesamtausgabe der Bayerischen Akademie der Wissenschaften, Stuttgart-Bad Cannstatt, Frommann-Holzboog, 1987 y ss., t. I.4, p. 118. 
Por un lado, el spinozismo es denunciado como una doctrina ateísta en tanto que simplemente niega la existencia de un Dios teísta, niega la divinidad de las Sagradas Escrituras y, por lo tanto, aniquila la religión revelada, la moral y el orden político fundados en una divinidad trascendente, justa, libre. El auténtico significado de la acusación de ateísmo es, en este primer sentido, el señalamiento de Spinoza como un peligro.

Si las tesis del TTP hicieron que muchos filósofo y teólogos, como Jacob Thomasius, Musaeus y Christian Kortholt, vieran en el spinozismo un peligro para el orden religioso y político, y denunciaran a Spinoza como un enviado del diablo con la misión de subvertir los derechos divinos y humanos para perjuicio de la Iglesia y el Estado, otros vieron que la peligrosidad de la doctrina spinoziana se fundaba en su afirmación de la necesidad absoluta, de un determinismo supuestamente ciego y, por tanto, lo denunciaron como un ateo por negar a Dios una voluntad libre. Este es el caso de Leibniz y Wolff así como de Dippel. Jacobi, por su parte, encarna la expresión más radical de esta actitud hacia Spinoza, pues para él el spinozismo -y toda la filosofía racionalista- aniquila la religión y la moral y por lo tanto pone en peligro el orden social y político. Su denuncia de ateísmo contra Spinoza apunta, pues, a remarcar las consecuencias inaceptables de la doctrina spinoziana.

De modo que la confrontación con la doctrina spinoziana tanto del TTP como del la Ética enfrenta a los teólogos y filósofos alemanes al problema de cuáles son las premisas metafísicas requeridas para la fundamentación y la conservación del orden político, religioso y moral. Así, la lucha contra el monismo ontológico y el determinismo naturalista spinozianos y su consecuente defensa de la democracia y de la posibilidad de un acceso personal a Dios, sin mediaciones por parte de ninguna Iglesia, pone en evidencia hasta qué punto estos pensadores del siglo XVIII vieron en el spinozismo un peligro para el orden.

Observar la cuestión de este modo permite a su vez comprender por qué ciertos personajes heterodoxos y enemigos del statu quo, como Edelmann o Schmidt encontraron en Spinoza un aliado para sus batallas y fueron perseguidos y condenados por ser spinozistas clandestinos. Sus diferentes recuperaciones del spinozismo podrían incluso ser vistas como una primera salvación de esta doctrina, aunque esta no implicaría un llamado 
a modificar la comprensión que se tenía de ella, sino simplemente a situarse en un mismo frente de batalla junto a Spinoza.

Pero además, Spinoza es denunciado como ateísta y rechazado por ciertos pensadores alemanes, ya no por lo que su doctrina niega ni por las consecuencias de esa negación, sino por el hecho de haber postulado una divinidad que, en vez de ser objeto de la fe, es demostrada racionalmente. Su noción de Dios pretende ser un producto de la razón humana, que se presenta como la máxima autoridad en la investigación de la verdad.

En tanto que su posición pretende ser racional y sistemática-Spinoza es el ateo de sistema, según la caracterización de Pierre Bayle- sus enemigos intentarán mostrar que es allí donde radica su error. Incluso atribuirán el hecho de que Spinoza afirme lo que ellos consideran como absurdos a cierta exageración en el uso de la razón demostrativa -el spinozismo es cartesianismo radicalizado, según la caracterización de Leibniz.

Este problema, el problema del alcance de la razón humana, constituye el núcleo de la polémica que Christian Thomasius y Walther von Tschirnhaus, así como el trasfondo de la acusación de spinozismo contra Wolff por parte de Joachim Lange. Este es el verdadero problema detrás del Spinozismusstreit entre Mendelssohn y Jacobi, y también Kant discute a Spinoza en este ámbito problemático: el spinozismo, en tanto que es una filosofía dogmática que se aventura con la razón especulativa más allá de los límites legítimos de su uso, conduce, según la visión kantiana, al ateísmo y al fanatismo.

De modo que este segundo aspecto problemático que subyace a la discusión del spinozismo en Alemania se superpone con el problema del poder y los límites de la razón, del derecho de la fe y el sentido de la revelación, cuyas implicancias llegan hasta la posibilidad de fundamentar de modo autónomo la moral, desligar lo político de poderes trascendentes y erigir una religión puramente racional, igual para todos los hombres. Así, a finales del siglo XVIII, posicionarse frente a Spinoza era posicionarse frente al problema del sentido y el alcance del proyecto de la Auflelärung, que en muchos aspectos parecía coincidir con las propuestas éticas y políticas de Spinoza ${ }^{64}$.

${ }^{64}$ Respecto de la relación entre el spinozismo y la Ilustración, véanse Jacob, 
En definitiva, es este segundo aspecto de la discusión acerca del spinozismo lo que motiva su santificación por parte de ciertos pensadores alemanes y el consiguiente renacimiento de su doctrina. Jacobi, quien denuncia a Spinoza como un ateo y un fatalista, está sin embargo dispuesto a llamarlo Heiliger Benedictus [Santo Benedictus], porque su figura representa la coherencia y la honestidad intelectual de la que, según él, Mendelssohn y los ilustrados berlineses carecían ${ }^{65}$. Goethe y Herder también ven en Spinoza un santo, pero el motivo y el resultado de esta santificación son muy diferentes a los de Jacobi. En primer lugar, podría decirse que coinciden con la posición jacobiana al afirmar que el spinozismo es para ellos el único sistema filosófico que ofrece un concepto de Dios coherente con la razón humana. Sin embargo, este Dios spinoziano -inmanente, natural, impersonal, necesario- no conduce para ellos al abismo del ateísmo y de la inmoralidad, sino que al contrario, es el Dios que hace posible una moral y una religión.

Nuestro análisis pone, pues, en evidencia que Spinoza fue acusado como un ateo por los pensadores alemanes, ya por lo que su doctrina niega -el Dios del teísmo, la revelación, el libre arbitrio- ya por lo que su doctrina afirma -el Dios inmanente y necesario- pero esto, especialmente, por cómo lo afirma: racional y sistemáticamente. Al ocuparse de la doctrina spinoziana, los pensadores alemanes debieron cuestionarse qué alcance estaban dispuestos a reconocerle a la razón humana y, paralelamente, si no debían admitir con Lessing que los conceptos ortodoxos de la divinidad ya no los satisfacían. Creemos que estas dos cuestiones permanecen latentes como trasfondo de las discusiones filosóficas de la última década del siglo XVIII en Alemania y que proveen una clave para comprender el lugar complejo que Spinoza ocupa en las obras de los autores que dan origen al Idealismo alemán.

Recibido: 09/2010; aceptado: 10/2010.

Margaret, The Radical Enlightenment: Pantheists, Freemasons, and Republicans, George Allen and Unwin, London, 1981; Israel, Jonathan I., Radical Enlightenment: Philosophy and the Making of Modernity 1650-1750, Oxford, Oxford University Press, 2001; Pätzold, op. cit.

${ }^{65} \mathrm{~F}$. H. Jacobi, Wider Mendelssobns Beschuldigungen, betreffend die Briefe über die Lebre des Spinoza en Friedrich Heinrich Jacobi's Werke, ed. cit., t. IV.2, p. 245. 